\title{
Implementation of Cell Zooming Technique to Optimize the Base Station Power
}

\author{
Payal Santosh Gundawar', Prabhakar D. Dorge ${ }^{2}$, Chetan S. Gode ${ }^{3}$
}

M.Tech Student, Department of Electronics and Tele-Communication, Yeshwantrao Chavan College of Engineering,

$$
\text { Nagpur, India }{ }^{1}
$$

Asst. Professor, Department of Electronics and Tele-Communication, Yeshwantrao Chavan College of Engineering,

$$
\text { Nagpur, India } 2,3
$$

\begin{abstract}
This paper investigate the problem regarding the minimization of the power of the base station by using the cell zooming technique. In this paper, we mainly focused on reducing the power consumed at the base station end, whereas most of the power minimization related work in the literature today is focused on the mobile end only. Cell zooming is a technology that promises to minimize the resource utilization. This technique is designed to save the energy consumed by the base station by maintaining the quality of services. To understand the working of the cell zooming techniques centralized algorithm is a very basic way. Our simulations shows that the proposed technique can achieve upto $25 \%$ reduction in the power consumption over the base station.
\end{abstract}

Keywords: Cell zooming, Traffic load, energy efficiency, base stations, bandwidth utilization, spectral efficiency, orthogonal frequency division multiplexing (OFDM) and power allocation.

\section{INTRODUCTION}

As we all know that mobile wireless communication is experiencing a tremendous growth today in terms of both the various number of users as well as the variety of applications coming day by day. As millions of new users are including each and every month. The mobile applications are changing from simple voice applications to data and than to gaming devices. To all of us, the word 'mobile' is very familiar, and its necessary to think much about its operation parts. As we all know that the mobile is very user friendly, that why it is not the case when it came into its operational cost. Communication is the process that should be maintained all the time and each of the base station is designed to operate $24 * 7$ hours. The number of base stations are deployed to the order of tens during the early decades. As most of the users are interested in data transfer rather than the voice transfer and therefore their is an increasing demand of the resources. Inorder to satisfy the users requirements the operator has to work hard as the users requirements are increasing day by day. The basic concepts regarding the cell zooming technique is very clearly explained in [3].

The detailed investigation is required regarding two issues they are data rate enhancement and the power optimization. Power optimization is one of the key issues for reasons that are both the economical and environmental in nature. As we all know that the industries are mostly focusing on the power optimization at the user equipment end only, while no one is focusing on the base station power optimization were it has been ignored. The important factor for customer satisfaction is its battery conservation at the user equipments but for this we can no longer ignore the power consumption at the base station. The base station power consumption is billions of dollars today according to some studies and therefore they will only increase in the future as the cellular service provider strive to increase their throughputs and widen their user base.

To optimize the base station power consumption their are various techniques that can be implemented are adaptive modulation, coding, opportunistic scheduling and the spectrum assignment. By using power optimization adaptive modulation schemes for single user links have been well understood. Their may be two forms bit loading or power loading of the adaptive modulation [4], [5]. If we consider the bit loading, the total number of bits are being distributed across the subcarriers that are being based on the channel state information (CSI). Which means that different number of bits are being carried by each subcarrier. Similarly, in case of power loading, the total power that is being available may be distributed across the subcarriers which is being based on the instantaneous value of the channel state information (CSI). Each of the subcarrier may consist of the different power. OFDM systems was proposed were the power loading for minimization of the symbol error probability has been considered [6], [7] and [8]. In order to distinguish such kind of the problems from the single-user power loading than researchers call that type of problem of power allocation. Subcarriers are providing diversity by interleaving with error control coding [9]-[11] This paper provide a solution for the problem regarding to the power optimization in the OFDM-based multiuser modems.

As we all know that the users are not properly served by the available number of base station for this we need to improve the service facility. To solve the problem the cellular companies have introduced more number of base stations inorder to fullfill the inflating user needs. The operational cost are also increasing as the cost for the resources such as coal, electricity are increasing. 
Moreover the resources are becoming so scarce day by day. Due to the uncontrolled user requirements the operators like to cut down the operational cost and the resource utilization and therefore they cannot cancel out the already deployed base stations. The only way to solve this problem are by 'improving the efficiency' or by 'reducing the resource consumption'. This paper shows the effect of cell zooming on energy saving. Depending upon the users density that means the number of users under each of the base station this type of base stations are selected for participating in the cell zooming algorithm. Whereas, energy conservation are maximized by increased number of sleeping base stations.

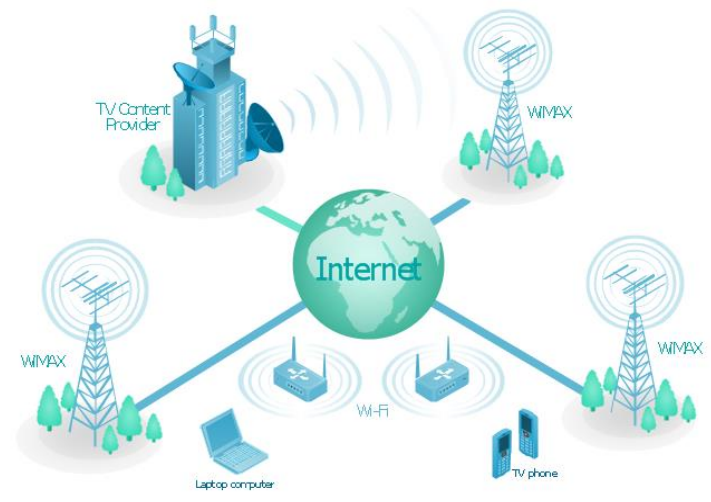

Fig. 1. Communication network of VANET.

Recently ever increasing interest in the vehicular ad hoc network communication necessitates takes place via different mobility pattern simulation including the vehicular performance study too. Here we have presented a study on the different mobility patterns for the large coverage area networks like wimax using NS2 which are being performed through various routing algorithm [12] In this paper we are going to improve the various parameter of the quality of service such as throughput, packet delivery ratio, packet loss ratio, jitter and delay. This is being performed for various routing algorithm with different mobility patterns for different networks.

\section{RELATED WORK}

The various steps that should be performed are shown below.

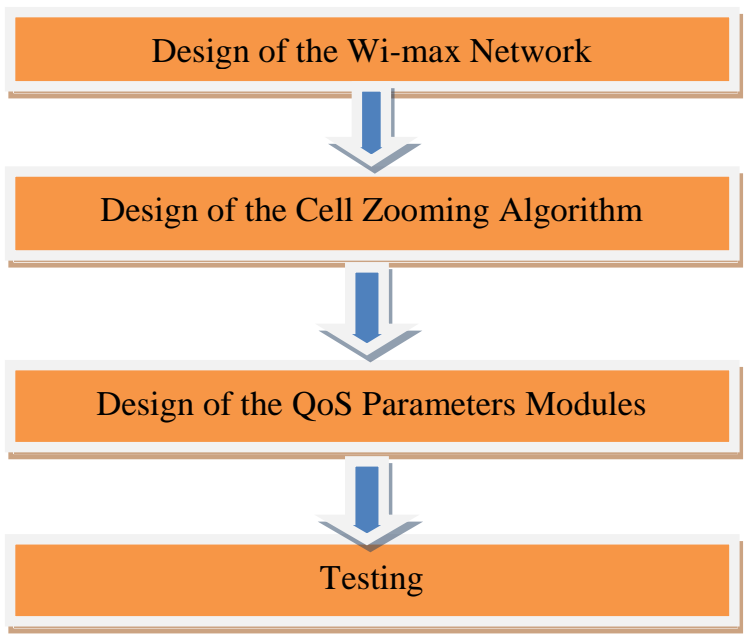

Fig. 2. Various steps for system designed.
A. Designing of the wi-max network :-

Firstly we need to design the wi-max network for which the various parameters are required and they are as follows;

Table 1. Parameters of the Designed System

\begin{tabular}{|c|c|c|}
\hline S.N. & Parameters & Protocols \\
\hline 1 & Routing Protocol & AODV \\
\hline 2 & Traffic Type & UDP \\
\hline 3 & Antenna & Omni directional antenna \\
\hline 4 & $\begin{array}{c}\text { Interface Queue } \\
\text { Type }\end{array}$ & Droptail \\
\hline 5 & $\begin{array}{c}\text { Radio } \\
\text { Propagation } \\
\text { Model }\end{array}$ & Two Ray Ground \\
\hline 6 & Packet Size & Wireless channel \\
\hline 7 & Channel Type & OFDM \\
\hline 8 & $\begin{array}{c}\text { Network } \\
\text { Interface }\end{array}$ & 50 \\
& $\begin{array}{c}\text { Maximum } \\
\text { Packets in } \\
\text { Interface Queue } \\
\text { Type }\end{array}$ & \\
\hline
\end{tabular}

B. Designing of the cell zooming algorithm :-

As we all know that the cell zooming is that which adaptively adjust the cell size according to the traffic load. We can consider cell zooming as a promising technique that assures us the minimization of the utilized resources. This system is designed to save whatever will be the energy consumed by the base station and simultaneously by maintaining the quality of services. As centralized algorithm is a very simple way through which we can understand how the cell zooming technique exactly works. Their are some advantages of cell zooming such as it is mainly used for two main purposes such as load balancing and energy saving. Load balancing is done by transfering whatever is the traffic from the cells under heavy load to the cells under light load. Whereas cell zooming can also be used for energy saving. Lets consider whatever will be the contary to the usage of the load balancing, then their the cells will zoom in to zero when light enough the traffic load is available. Whereas some of the base station works in the sleeping mode due to which the neighbour cell will zoom out according to whatever will be the guarantee of the coverage. Therefore we can say that cell zooming can perform both disperse the load for load balancing and as well as concentrate the load for energy saving. As we have studied that power control can help to ensure the efficient spatial reuse and as well as the minimization of the energy consumption. These type of the functionalities are similar to that of the cell zooming technique. Whereas cell zooming is different in many ways from that of the power control. Power control mainly focuses on the link level performance whereas cell zooming technique mainly focuses on the network level performance. In power control transmit power consumption is done whereas in cell zooming energy consumption of the whole network is done. Lastly, as we know that power control does not actively change its cell size whereas cell zooming is the one which actually 
changes the cell size by adjusting the transmit power of the control signal.

\section{SYSTEM DESIGN}

As we all know that the cell zooming is the technique which adaptively adjust the cell size according to the traffic load. Whereas cell zooming technique is to ensure the minimized power consumption by the base stations. Lets consider the centralized algorithm which is the very basic way to understand how exactly the cell zooming technique works.

It is one of the simplest algorithm [3] which help us to explain the cell zooming method. Figure below shows the flow chart that depict the complete process of the application that takes place. Depending upon the spectral efficiency of the base stations the user allocation is being done. First of all we need to compute the spectral efficiency of all the base stations. The base station with the highest spectral efficiency to that the user is being allocated. Depending upon the bandwidth constraint given by $L_{j} B_{j}+b_{i j} \leq$ idle $B_{j}$ we can determine whether a base station can serve a user or not. In this equation $L_{j}$ stands for the traffic load of the base station ' $\mathrm{j}$ '. $B_{j}$ stands for the total bandwidth of the base station and $b_{i j}$ stands for the individual user bandwidth. The user will be blocked if the constraint is not met. By considering the value of the ratio $R_{j}$ the base stations are ordered in the ascending manner after the users are being allocated. The base station can operate in the sleep mode when $R_{j}=0$ that means their are zero user under its coverage. Next, if it is not $R_{j}=0$ then we need to select the base station with the least ratio value. Reallocation of the users should be done before switching off the base station. By checking the bandwidth constraint re-association is performed. When all the users are re-associated the particular base station is switched off and if any of the user is blocked then the algorithm terminates.

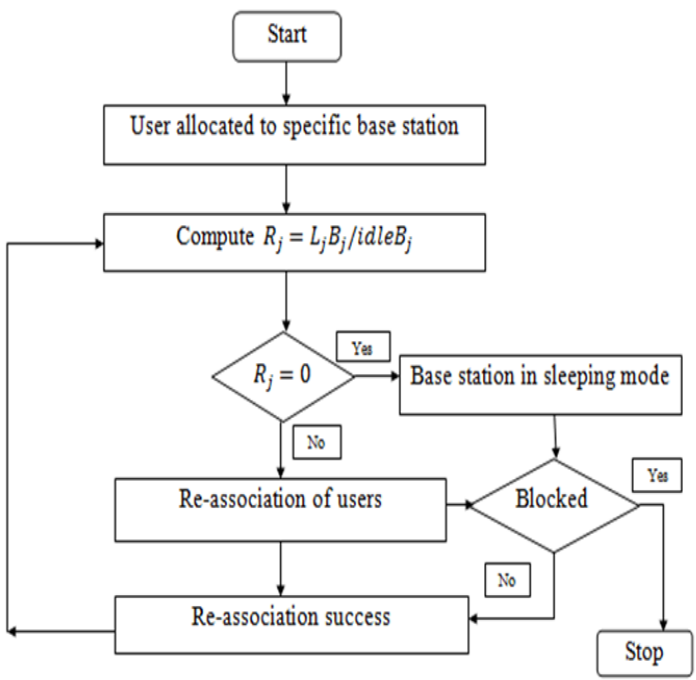

Fig. 3. Flow Chart for Cell Zooming Algorithm

C. Designing of the qos parameters modules :Their are various qos parameters modules such as jitter, throughput, delay, packet delivery ratio, packet loss ratio. (a) Jitter :- The jitter exactly mean that the time difference between the packets inter arrival time to their destination.

(b) Throughput :- Whatever will be the data delivered to all the nodes or terminals through out the network the sum of that data rates is known as system throughput.

(c) Delay :- The delay of the network is termed as how long it takes for a bit to travel over the network from one of the point to another. Whereas delay is measured in terms of fraction of seconds or multiples.

(d) Packet delivery ratio :- The amount of packets that are been successfully send from the source to the destination which is in future is further compared to that of the amount of packets that are being send out of the sender.

(e) Packet loss ratio :- Whatever will be the loss of packet that is one or more transmitted packets which is not been arrived to their destination that type of the ratio is known as packet loss ratio.

\section{SIMLATION RESULTS}

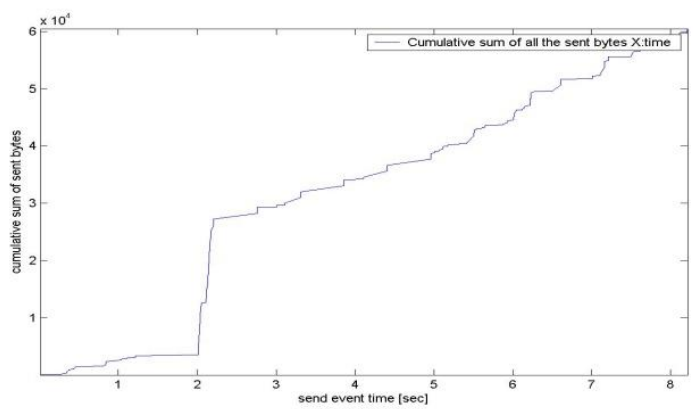

Fig. 4. Shows the cumulative sum of all the sent bytes $X$ in time.

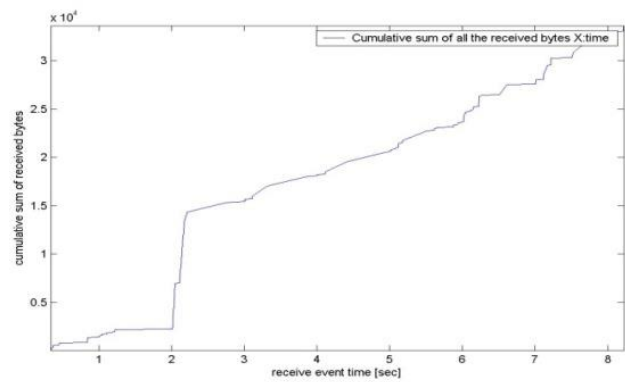

Fig. 5. Shows the cumulative sum of all the received bytes $\mathrm{X}$ in time.

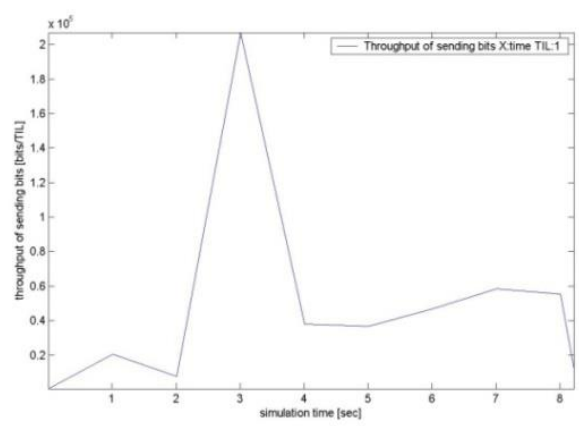

Fig. 6. Shows the throughput of sending bits $X$ in 


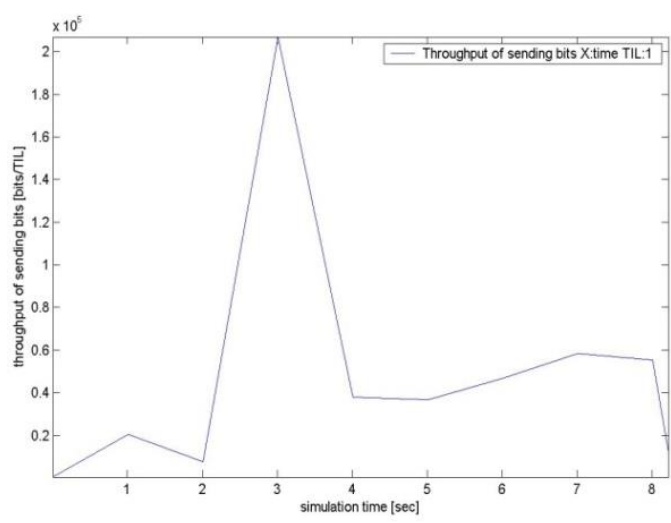

Fig. 7. Shows the throughput of the receiving bits $\mathrm{X}$ in time.

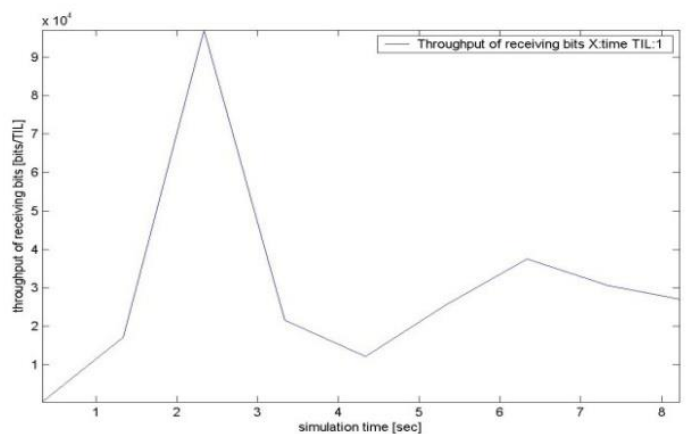

Fig. 8. Shows the jitter of all the sent packets in sequence.

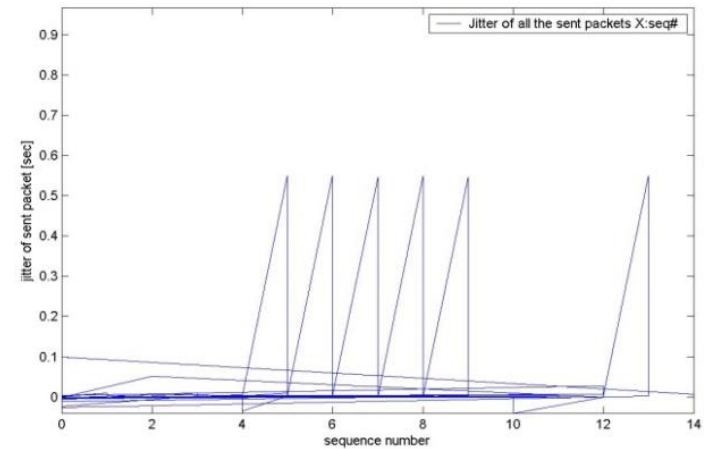

Fig. 9. Shows the jitter of all the sent packets in sequence

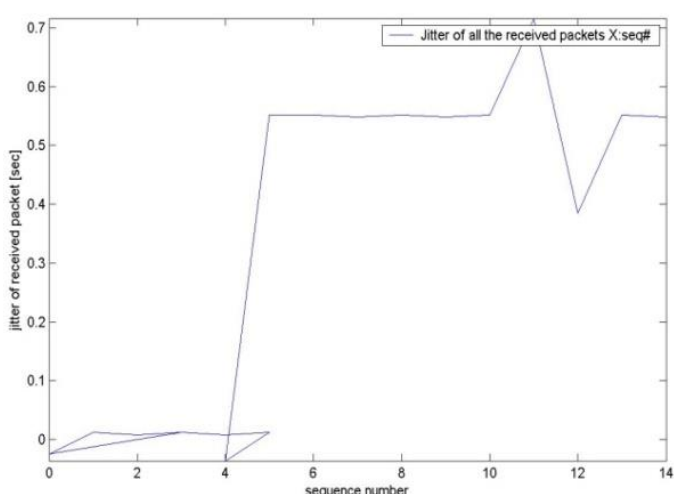

Fig. 10. Shows the jitter of all the received packets in sequence

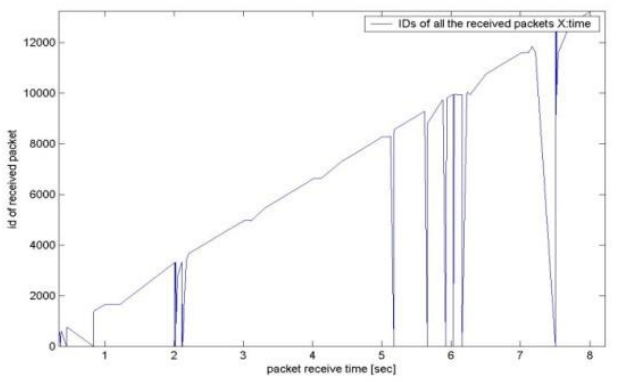

Fig. 11. Shows the Ids of all the received packets $X$ in time.

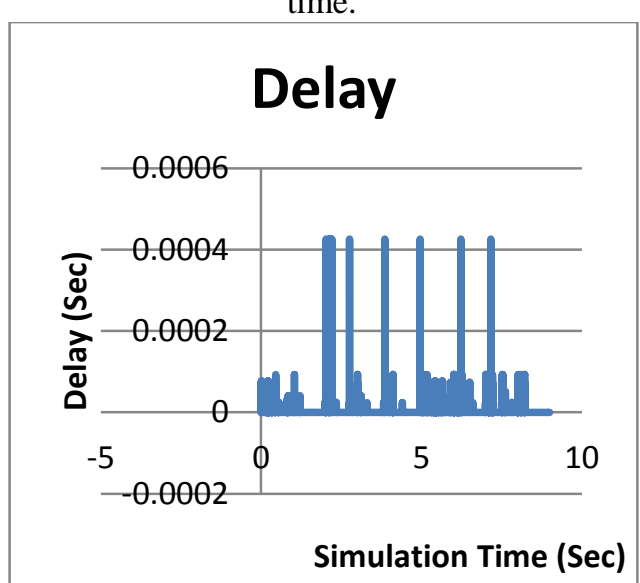

Fig. 12. Shows the graph for Delay

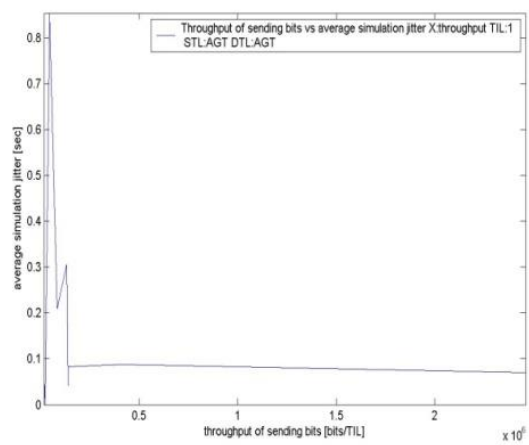

Fig. 13. Shows the throughput of sending bits vs average simulation of the jitter $X$.

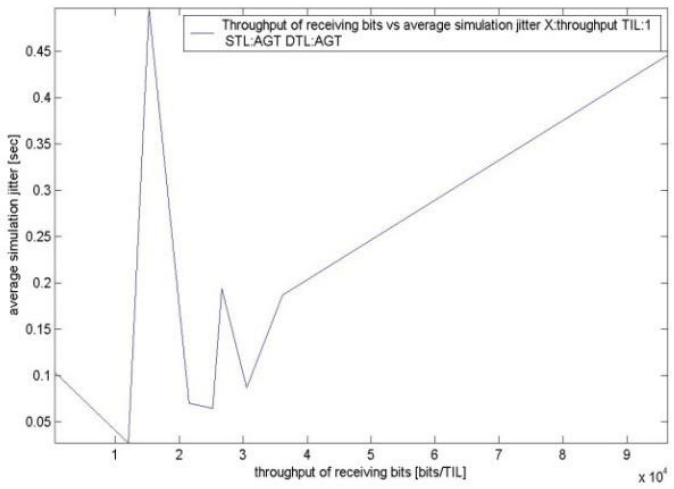

Fig. 14. Shows the throughput of receiving bits vs average simulation of jitter X. 
INTERNATIONAL JOURNAL OF INNOVATIVE RESEARCH IN ELECTRICAL, ELECTRONICS, INSTRUMENTATION AND CONTROL ENGINEERING Vol. 3, Issue 3, March 2015

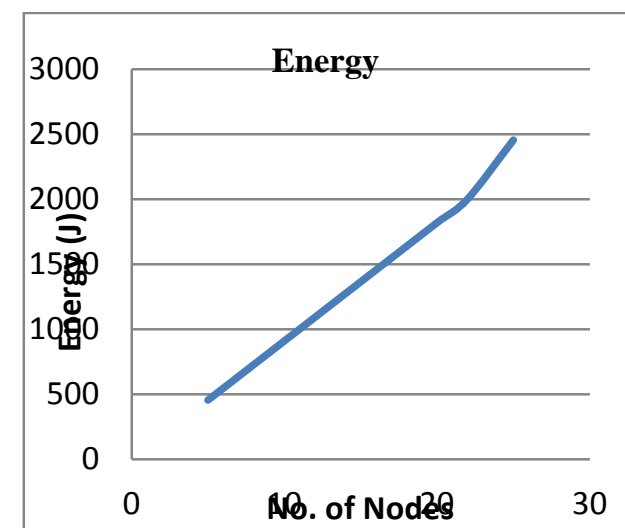

Fig. 15. Shows the graph for energy.

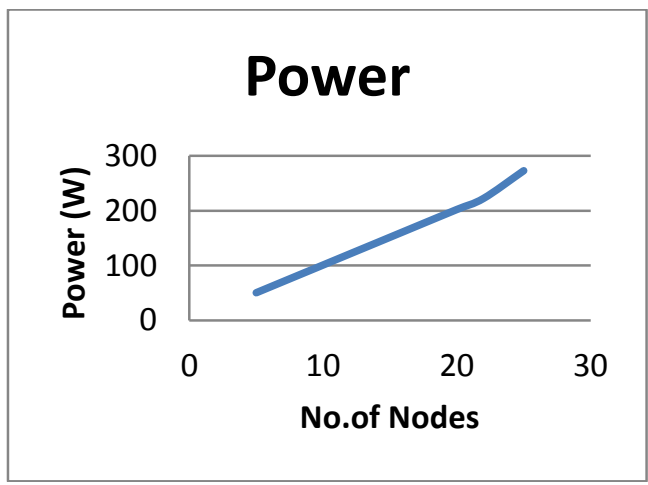

Fig. 16. Shows the graph for power.

Table 2. Shows values of the whole network.

\begin{tabular}{|c|c|}
\hline Quality of Service parameter & Output \\
\hline Average Throughput & $12882.8062 \mathrm{kbps}$ \\
\hline Power Delivery Ratio & $77.520584 \%$ \\
\hline Power Loss Ratio & $22.479416 \%$ \\
\hline Average Delay & $1.482 * 10^{-6} \mathrm{sec}$ \\
\hline
\end{tabular}

\section{CONCLUSION}

In this paper, we have proposed the system through which the base station end power is being minimized by an OFDM-based downlink cellular communication system. Further the cell zooming technique is being implemented in which the cells are adaptively adjusting according to the traffic load. As we all know that by switching off the base station the energy consumption is being minimized. Even from the tight scheduled of the cellular network it is clear that by implementing the cell zooming algorithm energy can be saved. When more number of the base stations are switched off the conservation of the energy is been increased. Thus, our simulations shows upto $25 \%$ reduction in the base station power consumption.

\section{REFERENCES}

[1] Santosh Nagaraj and Mahasweta Sarkar, "A Novel Power Allocation Technique for 4G Cellular Networks," 2014 International Conference on Computing, Networking and Communications, Wireless Communications Symposium

[2] Rinju Mariam Rolly,Poornima S. "Base Station Switching Based on Traffic Load Analysis "International Journal of Advance Foundation and Research in Computer (IJAFRC)Volume 1, Issue 6, June 2014.
[3] Zhisheng Niu, Yiqun Wu, Jie Gong, and Zexi Yang, Tsinghua University.,"Cell Zooming for Cost-Efficient Green Cellular Networks," IEEE Commun. Mag., vol. 48, issue.11, Nov. 2010, pp.74-79.

[4] S. T. Chung and A. Goldsmith, "Degrees of freedom in adaptive coding and modulation: A unified view," IEEE Trans. Commun., pp. 1561-1571, Sept 2001

[5] K.-B. Song, A. Ekbal, S. T. Chung, and J. Cioffi, "Adaptive modulation and coding (AMC) for bit interleaved coded OFDM (BIC-OFDM)," IEEE Trans. Wireless Commun., pp. 1685-1694, July 2006.

[6] L. Goldfeld, V. Lyandres, and D.Wulich, "Minimum BER power loading for OFDM in fading channel," IEEE Trans. Commun., pp. 1729-1733, Nov 2002.

[7] A. Marques, F. Digham, and G. Giannakis, "Optimizing power efficiency of OFDM using quantized channel state information," IEEE J. Sel. Areas Commun., pp. 1582-1592, Aug 2006.

[8] S. Nagaraj and M. Sarkar, "Adaptive modulation for limited diversity fading channels," in Proc. IEEE Veh Tech Conf, 2009, pp. 1-6.

[9] G. Caire, G. Taricco, and E. Biglieri, "Bit-interleaved coded modulation," IEEE Trans. Inf. Theory, pp. 927-946, May 1998.

[10] S. B. Wicker, Digital Communication and Error Control Systems for Storage, 1st ed. Prentice Hall, 1995.

[11] A. J. Viterbi and J. K. Omura, Principles of Digital Communication and Coding. McGraw-Hill, 1979.

[12] Prabhakar D. Dorge, Sanjay S. Dorle, Megha B. Chakole and Dhira K. Thote, "Improvement of QoS in VANET with Different Mobility Patterns," 2012 International Conference on Radar Communication and Computing

[13] R. Balasubramaniam, S. Nagaraj, M. Sarkar, P. Khaitan, and C. Paolini, "Cell zooming for power efficient base station operation," in Proc. IEEE intl. on Wireless Comm. and Mob. Comput. Conf., 2013, pp. 556-560.

[14] Rinju Mariam Rolly and Poornima Sabu, "Performance Analysis of Cell Zooming Network," International Journal of Research in Advent Technology, Vol.2, No.5, May 2014

[15] Payal Santosh Gundawar, Prabhakar D. Dorge, Chetan S. Gode "Optimization of Base Station Power Using Cell Zooming Technique " International Journal of Advent Research in Computer and Electronics (IJARCE) Vol. 2, No. 1, January 2015 